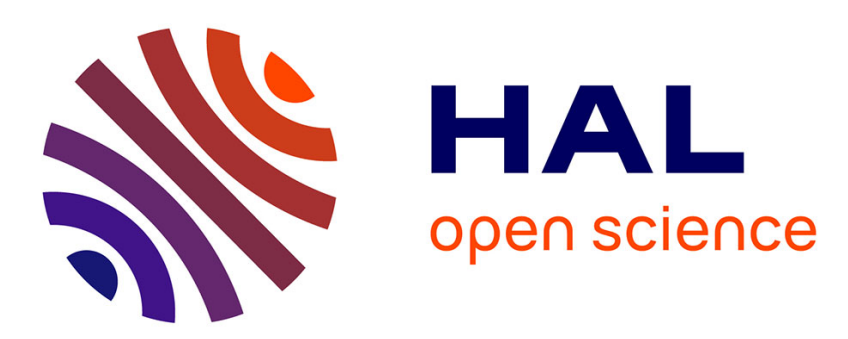

\title{
INDIRECT EXCHANGE MECHANISM OF MAGNETIC ORDERING IN AMORPHOUS ALLOYS
}

\author{
A. Madhukar
}

\section{To cite this version:}

A. Madhukar. INDIRECT EXCHANGE MECHANISM OF MAGNETIC ORDERING IN AMORPHOUS ALLOYS. Journal de Physique Colloques, 1974, 35 (C4), pp.C4-295-C4-298. 10.1051/jphyscol:1974455 . jpa-00215646

\section{HAL Id: jpa-00215646 https://hal.science/jpa-00215646}

Submitted on 1 Jan 1974

HAL is a multi-disciplinary open access archive for the deposit and dissemination of scientific research documents, whether they are published or not. The documents may come from teaching and research institutions in France or abroad, or from public or private research centers.
L'archive ouverte pluridisciplinaire HAL, est destinée au dépôt et à la diffusion de documents scientifiques de niveau recherche, publiés ou non, émanant des établissements d'enseignement et de recherche français ou étrangers, des laboratoires publics ou privés. 


\title{
INDIRECT EXCHANGE MECHANISM OF MAGNETIC ORDERING IN AMORPHOUS ALLOYS
}

\author{
A. MADHUKAR
}

IBM Thomas J. Watson Research Center

Yorktown Heights, N. Y. 10598, U. S. A.

\begin{abstract}
Résumé. - L'existence d'une longueur finie pour la cohérence de phase dans les matériaux amorphes entraîne un amortissement de la polarisation RKKY habituelle du spin des électrons de conduction.

La nature de l'ordre magnétique dépend des grandeurs relatives de cette longueur d'amortissement et de la séparation moyenne entre les atomes magnétiques. Bien que, dans la plupart des cas, une diminution de la température de Curie paramagnétique, $T_{\mathrm{e}}$, soit attendue (et observée), nous montrons qu'il existe aussi la possibilité d'une augmentation de $T_{\mathrm{e}}$ par rapport au cas cristallin.

Abstract. - The existence of finite phase coherence length in amorphous materials implies a damping of the usual RKKY conducting electron spin polarization. The nature of magnetic ordering depends upon the relative magnitudes of this decay length and the average separation of magnetic atoms. Although, in most cases a decrease in the paramagnetic Curie temperature, $T_{\mathrm{c}}$, is to be expected (and observed), we show that there also exists the possibility of an increase in $T_{\mathrm{c}}$, as compared to the crystalline case.
\end{abstract}

The magnetic behaviour of disordered and amorphous metallic materials has received considerable experimental attention in the recent past [1]. Due to lack of long range order, amorphous materials exhibit a vastly reduced paramagnetic Curie temperature, $T_{\mathrm{c}}$, as compared to the crystalline state [2]. In some instances, lack of detailed experimental knowledge of the structure has led to considerable speculation on the manner in which the magnetic atoms are distributed in the alloy. It has been suggested that a random distribution of impurities generally leads to zero $T_{\mathrm{c}}$ whereas a small but finite $T_{\mathrm{c}}$ is a reflection of clustering of magnetic atoms [3]. Although intuitively one expects a lowering in $T_{\mathrm{c}}$ with increasing disorder (or amorphous nature) the transformation of these intuitive feelings into a mathematical form has turned out to be a difficult task. Our lack of understanding of the nature of electronic states in amorphous materials has been a major stumbling block in understanding its magnetic properties. While the present fervent activity provides hope for a reliable description of the electronic states, in the meantime, we present here the simplest possible description of indirect exchange mechanism of magnetic ordering consistent with the requirement of a small phase coherence length in the amorphous state. It is the analogue of the Rudermann-Kittel-KasuyaYosida (RKKY) mechanism, well accepted and widely used for crystalline materials [4]. Since magnetic ordering is determined by the relation between the range of exchange interaction and the configuration of magnetic atoms, it becomes imperative to incorporate a finite mean free path, a quantity that severely affects the range of indirect exchange interaction. The absence of a long range structural order in amorphous materials not only makes useless the use of Bloch functions, but also fundamentally requires a mixing of various momentum states. It is well known that in the amorphous state there exist extended electronic states in the center of the band and localized states at the edges of the band. The major contribution to the electrical conductance comes from the extended states. The amorphous magnetic materials we are interested in here show metallic conductance [1]. As such, the nature of the extended states is crucial to the indirect magnetic ordering mechanism of the kind operative in these materials. A plane wave description of the electronic states implies long ranged (infinite to be precise) wave length and phase coherence. Such a description is incompatible with the nature of the extended states in amorphous materials. Consequently the standard RKKY expression for the conduction electron spin polarization, based on plane wave states no longer holds for amorphous materials. The simplest way to give the extended states a finite spatial extent is achieved by constructing linear combination of Bloch functions; $\varphi_{\bar{k}}(\bar{r})$;

$$
\psi(\bar{r})=\sum_{\bar{k}} A_{\bar{k}} \varphi_{\bar{k}}(\bar{r})
$$

where

$$
A_{\bar{k}}=\left\langle\varphi_{\bar{k}}(\bar{r}) \dot{\eta}^{\eta} \psi(\bar{r})\right\rangle
$$


Economou et al. [5] have conjectured that for weak scattering from randomly distributed scatterers, the coefficients $A_{k}$ are roughly constant in magnitude on a constant energy surface, but have their phases distributed randomly over different regions of linear dimension $\xi$; the phase coherence length. Thus random multiple scattering leads to an additional factor $\mathrm{e}^{-R / \xi}$ in the average autocorrelation function,

$$
\left\langle\psi^{*}(\bar{r}) \psi(\bar{r}+\bar{R})>\sim \mathrm{e}^{-R / \xi}\right.
$$

where the average is carried over an ensemble of imperfect crystals with a random distribution of imperfections.

On the other hand, the amorphous materials investigated in the context of magnetism show liquidlike $x$-ray diffraction patterns [6]. The Fermi surface is smeared by a small spread $\Delta k$ in the momentum space. A finite spread in momentum leads to a finite spatial extent $\Delta r \sim(\Delta k)^{-1}$ in the electronic states. If $\xi<\Delta r$, then the coefficients $A_{k}$ may be considered to have the same phase over the spatial extent $\Delta r$ with varying magnitude. In this case we may choose a Gaussian distribution for $\left(A_{k}\right)^{2}$ centered around $K_{0}$ with standard deviation $\Delta k$. Thus we write for the electrons,

$$
\psi_{\bar{k}_{0}}(\bar{r})=\left[2 \pi(\Delta r)^{2}\right]^{-3 / 4} \mathrm{e}^{\overline{i \bar{k}_{0} \cdot \bar{r}}} \mathrm{e}^{-1 / 4(\Delta k)^{2} \boldsymbol{r}^{2}} .
$$

The spatial extent of the electronic states may be related to the electron mean free path, $\lambda$. We do not attempt to find this relationship, but it suffices for our purposes to note that $\Delta r=a \lambda$, where $a$ is of the order of unity. Admittedly, the wave packet states (4) are not the real electronic states of the amorphous alloy. However, their use in describing the electronic motion in the context of indirect magnetic ordering mechanism can be justified by comparing the characteristic distance, $d_{\mathrm{c}}$, over which a typical wave packet of group velocity $v_{\mathrm{F}}$ can travel without much distortion and the average separation of magnetic atoms in the alloy, which is generally less than $10 \AA$. The characteristic time over which the wave does not significantly change its shape is, $t_{\mathrm{c}} \sim 2 m_{\mathrm{c}}^{*}(\Delta r)^{2}$, where $\Delta r$ is the spread of the wave packet. Thus $d_{\mathrm{c}} \sim 2 m_{\mathrm{e}}^{*} v_{\mathrm{F}}(\Delta r)^{2}$. Taking $\Delta r$ to be the typical mean free path value for these alloys, $\sim 5 \AA$, we find $d_{\mathrm{c}} \sim 100 \AA$. Thus the use of wave packets may not be an unrealistic description of the electronic motion over magnetic atom separation distances.

In either of the two cases discussed above, we can anticipate a rapid damping of the electronic polarization. This destruction of the long ranged nature of the electron spin polarization leads to a weaker coupling with neighboring magnetic atoms and hence, a significantly reduced paramagnetic Curie temperature. Furthermore, another kind of smearing of the oscillations in electron spin polarization comes from an averaging over the positions of the atoms, utilizing the radial distribution function. We perform this averaging at the very end of the calculations.

1. Polarization. - The interaction of the electronspin system is written as,

$$
H=-\sum_{j} \int \mathrm{d} \bar{r} J_{\mathrm{ex}}\left(\bar{r}-\bar{R}_{j}\right) \bar{\sigma}(\bar{r}) \cdot \bar{S}\left(\bar{R}_{j}\right) \delta\left(\bar{r}-\bar{R}_{j}\right)
$$

where, for simplicity we have assumed a contact interaction. $r$ and $R_{j}$ refer to the electron and spin coordinates. The impurity spins polarize the conduction electrons, this polarization in turn interacting with the neighboring spins. To calculate the nature of the electron spin polarization, we write (5) in terms of electron local creation and annihilation operators to obtain,

$$
\begin{aligned}
H=- & J_{\mathrm{cx} x}(0) \sum_{j}\left\{\left(b_{\uparrow}^{+}\left(\bar{R}_{j}\right) b_{\uparrow}\left(\bar{R}_{j}\right)-b_{\downarrow}^{+}\left(\bar{R}_{j}\right) b_{\downarrow}\left(\bar{R}_{j}\right)\right) \times\right. \\
& \times S_{z}\left(\bar{R}_{j}\right)+b_{\uparrow}^{+}\left(\bar{R}_{j}\right) b_{\downarrow}\left(\bar{R}_{j}\right) S_{-}\left(\bar{R}_{j}\right) \\
& \left.+b_{\downarrow}^{+}\left(\bar{R}_{j}\right) b_{\uparrow}\left(\bar{R}_{j}\right) S_{+}\left(\bar{R}_{j}\right)\right\}
\end{aligned}
$$

where $b_{\uparrow}^{+}\left(\bar{R}_{j}\right)$ creates an electron with spin up at $\bar{R}_{j}$, etc. Further, in accordance with the remarks on the nature of electronic states made in the introduction, we expand these local creation and annihilation operators in a basis set of electronic states which we denote by $\varphi_{\alpha}$. Thus,

$$
\begin{aligned}
& b_{\sigma}\left(\bar{R}_{j}\right)=\sum_{\alpha} \varphi_{\alpha}\left(\bar{R}_{j}\right) a_{\alpha \sigma} \\
& b_{\sigma}^{+}\left(\bar{R}_{j}\right)=\sum_{\alpha} \varphi_{\alpha}^{*}\left(\bar{R}_{j}\right) a_{\alpha \sigma}^{+} .
\end{aligned}
$$

For a crystalline system $\varphi_{\alpha}$ is chosen to be Bloch waves. For an amorphous system we shall use wave packet states for $\varphi_{\alpha}$. Using (7) we obtain from (6),

$$
\begin{aligned}
H= & -J_{\mathrm{ex}}(0) \sum_{\alpha \alpha^{\prime}} \varphi_{\alpha}^{*}\left(\bar{R}_{j}\right) \varphi_{\alpha^{\prime}}\left(R_{j}\right)\left\{\left(a_{\alpha \uparrow}^{+} a_{\alpha^{\prime} \uparrow}-a_{\alpha \downarrow}^{+} a_{\alpha^{\prime} \downarrow}\right)\right. \\
& \left.\times S_{z}\left(\bar{R}_{j}\right)+a_{\alpha \uparrow}^{+} a_{\alpha^{\prime} \downarrow} S_{-}\left(\bar{R}_{j}\right)+a_{\alpha \downarrow}^{+} a_{\alpha^{\prime} \uparrow} S_{+}\left(\bar{R}_{j}\right)\right\} .
\end{aligned}
$$

We use (8) to work out the conduction electron spin polarization by calculating the modification of the electron state $\varphi_{a}$ in first Born approximation. The method is straight forward and widely known [7]. The result is,

$$
\begin{aligned}
& p(\bar{r})=\rho_{\dagger}(\bar{r})-\rho_{\downarrow}(\bar{r}) \\
& =\left[-2 J_{\mathrm{ex}}(0) \sum_{j} \sum_{\alpha \alpha^{\prime}} \frac{S_{z}\left(\bar{R}_{j}\right)}{E_{\alpha}-E_{\alpha^{\prime}}} \times\right. \\
& \quad \times\left\{\varphi_{\alpha}^{*}\left(\bar{R}_{j}\right) \varphi_{\alpha}(\bar{r}) \varphi_{\alpha^{\prime}}^{*}(\bar{r}) \varphi_{\alpha^{\prime}}\left(\bar{R}_{j}\right)\right. \\
& \left.\left.+\varphi_{\alpha}^{*}(\bar{r}) \varphi_{\alpha}\left(\bar{R}_{j}\right) \varphi_{\alpha^{\prime}}^{*}\left(\bar{R}_{j}\right) \varphi_{\alpha^{\prime}}(\bar{r})\right\}\right]
\end{aligned}
$$

where $\rho_{\uparrow}, \downarrow(r)$ are the conduction electron density for spin up and spin down and their difference is the spin polarization $p(r)$.

To evaluate expression (9) for $p(r)$ we need to make 
statements about the nature of the states $\varphi_{\alpha}$, the energies $E_{\alpha}$ and the electron occupation number $n_{\alpha}$. It would be recalled that for the crystalline case these are the Bloch functions, the free electron energies with some effective mass and the Fermi function respectively. With these substitutions one obtains the well known RKKY polarization which has a long ranged oscillatory nature. The long ranged effects are related only to the density of states and their derivatives at the Fermi energy. In the present case, a finite spatial extent of the states $\varphi_{\alpha}$ shall significantly modify this long ranged nature.

Let us first consider the case of weak disorder. The sum over $j$ in (9) which is to be interpreted as an average over the random distribution of atoms may be approximated by,

$$
\begin{aligned}
\left\langle\varphi_{\alpha}^{*}\left(\bar{R}_{j}\right) \varphi_{\alpha}(\bar{r}) \varphi_{\alpha^{\prime}}^{*}(\bar{r}) \varphi_{\alpha^{\prime}}\left(\bar{R}_{j}\right)\right\rangle \simeq \\
\simeq<\varphi_{\alpha}^{*}\left(\bar{R}_{j}\right) \varphi_{\alpha}(\bar{r})>\left\langle\varphi_{\alpha^{\prime}}^{*}(r) \varphi_{\alpha^{\prime}}\left(\bar{R}_{j}\right)>\right. \\
\sim \mathrm{e}^{-2\left|\bar{r}-\bar{R}_{j}\right| / \xi}
\end{aligned}
$$

where we have used (3) in the last step. Thus it is apparent that the polarization $p(r)$ in this case shall have an exponentially decaying factor, reducing its range considerably.

For amorphous conducting materials showing diffusive metallic conductivity, we use (4) for the states $\varphi_{\alpha}$ as discussed in section 1. Further, we approximate $E_{\alpha}$ 's by the mean kinetic energy $\left(\hbar^{2} k_{0}^{2} / 2 m\right)$, of free wave packet states of the type (4). Finally, we may allow for a spread $\Delta k$ around Fermi momentum in the occupation function $n_{k}$. However, the spread in $n_{k}$ modifies only slightly the oscillatory nature of the polarization. For the purposes of magnetic ordering it is the exponential damping factor that is of importance in fixing the range of interaction. Therefore neglecting the spread in $n_{k}$, we obtain from (9),

$$
\begin{aligned}
p(\bar{r})=\frac{-2 J_{\mathrm{ex}}(0)}{E_{\mathrm{F}}} & q \pi\left(\frac{N e}{N_{\mathrm{A}}}\right)^{2} \sum_{j}\left[F\left(2 k_{\mathrm{F}}\left|\bar{r}-\bar{R}_{j}\right|\right) \times\right. \\
& \left.\times \exp \left\{-\frac{1}{2} \frac{r^{2}+R_{j}^{2}}{(\Delta r)^{2}}\right\}\right],
\end{aligned}
$$

$F(x)=x^{-4}(x \cos x-\sin x)$.

The rapid decay of the polarization in amorphous metals severely restricts the range of the indirect exchange between magnetic atoms. This weaker coupling in turn leads to a reduced Curie temperature and magnetization, as we show next.

2. Effective Heisenberg interaction. - The interaction of the polarization $p(r)$ with other impurity spin gives rise to an effective spin-spin interaction of the Heisenberg form, which in the standard way [4] is given by,

$$
H_{\text {spin-spin }}=-\sum_{i \neq j} I_{i j} \vec{S}_{i} \cdot \bar{S}_{j}
$$

where,

$$
\begin{aligned}
I_{i j}=\frac{2 J_{\mathrm{ex}}^{2}}{E_{\mathrm{F}}} q \pi z^{2} F\left(2 k_{\mathrm{F}}\left|\bar{R}_{i}-\bar{R}_{j}\right|\right) \times \\
\times \exp \left\{-\frac{1}{2} \frac{R_{i}^{2}+R_{j}^{2}}{(\Delta r)^{2}}\right\} .
\end{aligned}
$$

The effective indirect interaction (12) leads to the following expression for the paramagnetic Curie temperature, in the standard mean field approximation,

$$
T_{\mathrm{c}}=c \frac{J(J+1)(g-1)^{2}}{3 k_{\mathrm{B}}} \sum_{i \neq j} I_{i j},
$$

where $c=\left(N_{\mathrm{I}} / N_{\mathrm{A}}\right)$ is the magnetic atom concentration and we have replaced the spin $S$ by the total angular momentum $J$ which is a good quantum number for rare earth materials. To see the interplay of the range of effective interaction and the distribution of magnetic atoms, we introduce the pair correlation function in (4) to obtain :

$$
\begin{aligned}
I_{\mathrm{c}}=c & \frac{J(J+1)(g-1)^{2}}{3 k_{\mathrm{B}}} \frac{2 J_{\mathrm{ex}}^{2}}{E_{\mathrm{F}}} q \pi z^{2} \times \\
& \times 4 \pi \iint F\left(2 k_{\mathrm{F}} R_{i j}\right) R_{i j}^{2} g\left(R_{i j}\right) \\
& \times \exp \left[-\frac{1}{2}\left(\frac{R_{i}^{2}+R_{j}^{2}}{\lambda^{2}}\right)\right] \mathrm{d} R_{i} \mathrm{~d} R_{j} .
\end{aligned}
$$

From (15) it is clear that the magnitude and sign of the integral characteristically depends upon the position of the peaks of the radial aistribution function relative to the damped oscillatory nature of the conduction electron polarization. In general, the reduction in the effective strength of the interaction due to the decay factor shall lead to a reduction in the paramagnetic Curie temperature. This is in agreement with most experimental results. However, it must be noted that there is also a possibility of an increase in $T_{c}$, if for the amorphous state the pair correlation function happens to have a nearest neighbour peak positioned more favorably in the polarization of the electrons, as opposed to the crystalline state. This leads to the interesting phenomenon of piezomagnetism, already noted in the literature [8]. Indeed, the possibility of having magnetism in the amorphous state, even when the crystalline counterpart did not show any magnetic ordering, was pointed out by Gubanov a number of years ago [9]. (No experimental evidence, to the best of author's knowledge, has as yet been found for the existence of such amorphous magnetically ordered materials.) The possibility of a change of sign in (15), even with the damping factor, exists, and is a reminder of the possibility of ferromagnetic to antiferromagnetic transition brought about by varying the concentration of magnetic atoms. However, the damping factor shall not allow unphysical oscillations in $T_{\mathrm{c}}$ with varying $k_{\mathrm{F}}$, as is characteristic of the standard RKKY expression [4]. 
Although in the foregoing we have implicitly considered only one type of magnetic atom, it is clearly seen that the situation can be easily generalized to systems with more than one type of magnetic atom by making the values of $J_{\text {ex }}$ and spin depend upon the position and using the probability distributions for these magnetic atoms. Such a generalization would be needed for a comparison with such systems as $\mathrm{TbFe}_{2}$ (Rhyne et al., ref. [2]). Also it is believed that for this system, the only known material available in amorphous and crystalline states for the same composition, each spin experiences a strong local anisotropy field of random orientation. Thus a detailed study of the behavior of $\mathrm{TbFe}_{2}$ should incorporate an indirect exchange interaction of the type obtained in the present study and an anisotropy field.

Acknowledgment. - The author is thankful to R. Hasegawa for helpful discussions.

\section{References}

[1] Tsuer, C. C., in Amorphous Magnetism, ed. H. O. Hooper and A. M. de Graaf (Plenum Press) 1973, p. 299.

HASEGAWA, R., ibid, p. 309.

[2] Korn, D., Z. Phys. 214 (1968) 136.

RHXNe, J. J., et al., Phys. Rev. Lett. 29 (1972) 1562.

[3] KoK, W. C. and Anderson, P. W., Phil. Mag. 24 (1971) 1171.

[4] See, e. g., Matris, D. C., The Theory of Magnetism (Harper and Row, N. Y.) 1965.
[5] ECONOMOu, E. N. et al. in Amorphous and Liquid Semiconductors, ed. J. Tauc (Plenum Press) 1973.

[6] Hasegawa, R., Sol. Stat. Phys. Japan 5 (1970) 63.

[7] Yosida, K., Phys. Rev. 106 (1957) 893.

[8] Anderson. P. W., in Solid State Physics 14, (1963) 19 (Academic Press, New York).

[9] Gubanov, A. I., Quantum Electron Theory of Amorphous Conductors (Consultants Bureau, New York) 1965. 